PROCEEDINGS OF THE

AMERICAN MATHEMATICAL SOCIETY

Volume 138, Number 3, March 2010, Pages 965-973

S 0002-9939(09)10093-X

Article electronically published on October 19, 2009

\title{
A SUFFICIENT CONDITION FOR BIFURCATION IN RANDOM DYNAMICAL SYSTEMS
}

\author{
XIAOPENG CHEN, JINQIAO DUAN, AND XINCHU FU
}

(Communicated by Yingfei Yi)

\begin{abstract}
Some properties of the random Conley index are obtained, and then a sufficient condition for the existence of abstract bifurcation points for both discrete-time and continuous-time random dynamical systems is presented. This stochastic bifurcation phenomenon is demonstrated by a few examples.
\end{abstract}

\section{INTRODUCTION}

The Conley index is a topological tool for investigating dynamical systems 4, 10, 13, 14. In particular, the Conley index has been used to detect bifurcation in deterministic dynamical systems [2, 6, 7, 8, 9, 11. Recently, the Conley index was defined for discrete-time random dynamical systems [12. In the present paper, we investigate bifurcation for both discrete-time and continuous-time random dynamical systems, via the random Conley index.

We first present a few properties of the random Conley index in $\S 2$. Then in $\S 3$, we introduce a concept of prime random isolated invariant sets. Finally in $\S 4$, we obtain a sufficient condition for the existence of abstract bifurcation points for random dynamical systems.

\section{Some Properties of The RANDom Conley indeX}

A continuous random dynamical system (RDS) in the state space $X$, with the time set $\mathbb{T}$ and the underlying probability space $(\Omega, \mathcal{F}, \mathbb{P})$, consists of two ingredients [1:

(i) A model of the noise, namely a driving flow $\left(\theta_{t}\right)_{t \in \mathbb{T}}$ on the sample space $\Omega$, such that $(t, \omega) \mapsto \theta_{t} \omega$ is a measurable flow that leaves $\mathbb{P}$ invariant, i.e. $\theta_{t} \mathbb{P}=\mathbb{P}$ for all $t \in \mathbb{T}$.

(ii) A model of the system evolution influenced by noise, namely a cocycle $\phi$ over $\theta$, i.e., a measurable mapping $\phi: \mathbb{T} \times \Omega \times X \rightarrow X,(t, \omega, x) \mapsto \phi(t, \omega, x)$, such that

Received by the editors April 19, 2009, and, in revised form, June 24, 2009.

2000 Mathematics Subject Classification. Primary 37H20, 37B30, 60H10.

Key words and phrases. Random dynamical systems, discrete-time and continuous-time dynamical systems, random homeomorphism, Conley index, abstract bifurcation point.

The first author would like to thank Zhenxin Liu for helpful comments.

The second author was supported in part by NSF Grant 0620539, the Cheung Kong Scholars Program and the K. C. Wong Education Foundation.

The third author was supported in part by NSFC Grant 10672146.

(C)2009 American Mathematical Society Reverts to public domain 28 years from publication 
$(t, x) \mapsto \phi(t, \omega, x)$ is continuous for all $\omega \in \Omega$ and the family $\phi(t, \omega, \cdot)=\phi(t, \omega)$ : $X \rightarrow X$ of random mappings satisfies the cocycle property:

$$
\phi(0, \omega)=i d_{X}, \quad \phi(t+s, \omega)=\phi\left(t, \theta_{s} \omega\right) \circ \phi(s, \omega) \text { for all } t, s \in \mathbb{T}, \omega \in \Omega
$$

where $i d_{X}$ is the identity map on the state space $X$. We usually say that $\phi$ is a random dynamical system (over $\theta$ ). In this paper, the time set $\mathbb{T}=\mathbb{Z}$ or $\mathbb{R}$, and the state space $X$ is a locally compact complete metric space (e.g., $\mathbb{R}^{n}$ ). It follows from (2.1) that $\phi(t, \omega)$ is a homeomorphism of $X$ and

$$
\phi(n, \omega)^{-1}=\phi\left(-n, \theta_{n} \omega\right) .
$$

When $\phi$ is a discrete random dynamical system and $\varphi$ is the time-one map of $\phi$, i.e. $\varphi(\omega)=\phi(1, \omega): X \rightarrow X$, then we call $\varphi$ the random homeomorphism determined by $\phi$. On the other hand, if $\varphi$ is a random homeomorphism, then it generates via iteration a discrete $\operatorname{RDS} \phi(n, \omega, x)$. So we identify the discrete $\operatorname{RDS} \phi$ as a random homeomorphism.

The concept of topological equivalence of RDS is adapted from the deterministic case [1, 3]. Let $\phi_{1}$ and $\phi_{2}$ be two RDS over the same driving flow $\theta$, but with state spaces $X_{1}$ and $X_{2}$ respectively. The RDS $\phi_{1}$ and $\phi_{2}$ are said to be topologically equivalent if there exists a mapping $\alpha: \Omega \times X_{1} \rightarrow X_{2}$ with the following properties:

(i) The mapping $x \rightarrow \alpha(\omega, x)$ is a homeomorphism from $X_{1}$ onto $X_{2}$ for $\omega \in \Omega$.

(ii) Both mappings $\omega \rightarrow \alpha\left(\omega, x_{1}\right)$ and $\omega \rightarrow \alpha^{-1}\left(\omega, x_{2}\right)$ are measurable for $x_{1} \in$ $X_{1}$ and $x_{2} \in X_{2}$.

(iii) The cocycles $\phi_{1}$ and $\phi_{2}$ are cohomologous; i.e.,

$$
\phi_{2}(t, \omega, \alpha(\omega, x))=\alpha\left(\theta_{t} \omega, \phi_{1}(t, \omega, x)\right) \text { for } x \in X_{1} \text { and } \omega \in \Omega .
$$

We first recall some basic definitions from [12. A random compact set $N(\omega)$ is called a random isolating neighborhood if it satisfies

$$
\operatorname{InvN}(\omega) \subset \operatorname{int} N(\omega)
$$

where $\operatorname{int} N(\omega)$ denotes the interior of $N(\omega)$ and

$$
\operatorname{Inv} N(\omega)=\left\{x \in N(\omega) \mid \phi(n, \omega, x) \in N\left(\theta_{n} \omega\right), \forall n \in \mathbb{Z}\right\} .
$$

We call $S(\omega)$ a random isolated invariant set if there exists a random isolating neighborhood $N(\omega)$ such that $S(\omega)=\operatorname{Inv} N(\omega)$. A random compact set $N(\omega)$ is called a random isolating block if it satisfies $\varphi\left(\theta_{-1} \omega, N\left(\theta_{-1} \omega\right)\right) \cap N(\omega) \cap \varphi^{-1}(\theta \omega, N(\theta \omega))$ $\subset \operatorname{int} N(\omega)$.

For a random set $N(\omega)$, we define its exit set to be $N^{-}(\omega):=\{x \in N(\omega) \mid$ $\varphi(\omega, x) \notin \operatorname{int} N(\theta \omega)\}$.

We now introduce the concept of a random filtration pair as the random counterpart of the deterministic concept [5].

Definition 2.1 (Random filtration pair). Assume that $N(\omega)$ is a random isolating neighborhood, $L(\omega) \subset N(\omega)$ is a random compact set and $S(\omega)$ is a random isolated invariant set inside $N(\omega)$. Assume further that $N(\omega)=\operatorname{cl}(\operatorname{int} N(\omega))$ and $L(\omega)=$ cl $(\operatorname{int} L(\omega))$. We call $(N(\omega), L(\omega))$ a random filtration pair for $S(\omega)$ if the following conditions hold:

(i) $\operatorname{cl}(N(\omega) \backslash L(\omega))$ is a random isolating neighborhood of $S(\omega)$;

(ii) $L(\omega)$ is a random neighborhood of $N^{-}(\omega)$ in $N(\omega)$; and

(iii) $\varphi(\omega, L(\omega)) \cap \operatorname{cl}(N(\theta \omega) \backslash L(\theta \omega))=\emptyset$. 
Assume that $P=(N(\omega), L(\omega))$ is a random filtration pair for $S(\omega)$. Let $N_{L}(\omega)$ be the random quotient space (or random pointed space) $N(\omega) / L(\omega)$, where $N_{L}(\omega)$ $=(N(\omega) \backslash L(\omega) \cup[L(\omega)],[L(\omega)])$ for all $\omega \in \Omega$. If $L(\omega)=\emptyset$, then $N_{L}(\omega)=N(\omega) \cup[\emptyset]$. A map $\varphi_{P}(\omega, \cdot): N_{L}(\omega) \rightarrow N_{L}(\theta \omega)$ is called a random pointed space map associated to $P$ if

$$
\varphi_{P}(\omega, \cdot)= \begin{cases}{[L(\theta \omega)],} & x=[L(\omega)] \quad \text { or } \quad \varphi(\omega, x) \notin N(\theta \omega), \\ p(\theta \omega, \varphi(\omega, x)), & \text { otherwise, }\end{cases}
$$

where $p(\omega, \cdot): N(\omega) \rightarrow N_{L}(\omega)$ is the random quotient map. Assume that $C$ and $D$ are two random pointed spaces and that $c$ and $d$ are two Caratheodory functions (i.e., $c(\omega, \cdot), d(\omega, \cdot)$ are continuous and $c(\cdot, x), d(\cdot, x)$ are measurable) defined as

$$
c(\omega, \cdot): C(\omega) \rightarrow C(\theta \omega), \quad d(\omega, \cdot): D(\omega) \rightarrow D(\theta \omega) .
$$

Assume that $c$ and $d$ preserve base points. Two random pointed spaces $(C, c)$ and $(D, d)$ are called random shift equivalent and are denoted by $(C, c) \sim(D, d)$ if there exist random maps $r(\omega, \cdot): C(\omega) \rightarrow D\left(\theta_{n_{1}} \omega\right), s(\omega, \cdot): D(\omega) \longrightarrow C\left(\theta_{n_{2}} \omega\right)$ with measurable $n_{1}=n_{1}(\omega)$ and $n_{2}=n_{2}(\omega)$ such that they preserve base points and the following diagrams are quasi-commutative:

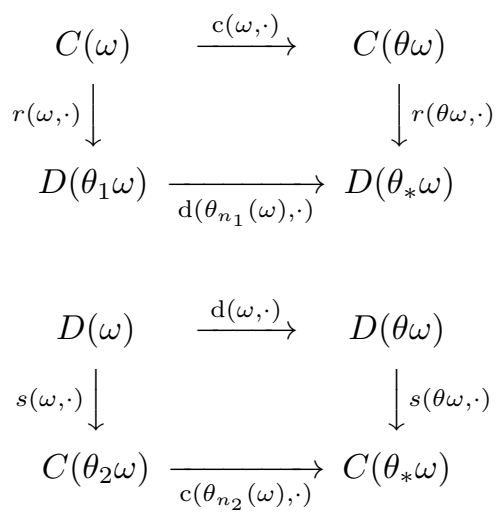

and the following hold:

$$
\begin{aligned}
& r\left(\theta_{n_{2}} \omega, s(\omega, \cdot)\right)=d^{n_{2}(\omega)+n_{1}\left(\theta_{n_{2}} \omega\right)}(\omega, \cdot), \\
& s\left(\theta_{n_{1}} \omega, r(\omega, \cdot)\right)=c^{n_{1}(\omega)+n_{2}\left(\theta_{n_{1}} \omega\right)}(\omega, \cdot) .
\end{aligned}
$$

Here "quasi-commutative" in the diagram (2.3) is in the sense that

$$
\left\{\begin{array}{l}
r(\theta \omega, c(\omega, \cdot))=d^{n_{1}(\theta \omega)-n_{1}(\omega)}\left(\theta_{n_{1}(\omega)+1} \omega, d\left(\theta_{n_{1}(\omega)} \omega, r(\omega, \cdot)\right)\right), n_{1}(\theta \omega) \geq n_{1}(\omega) \\
d^{n_{1}(\omega)-n_{1}(\theta \omega)}\left(\theta_{n_{1}(\theta \omega)+1} \omega, r(\theta \omega, c(\omega, \cdot))\right)=d\left(\theta_{n_{1}(\omega)} \omega, r(\omega, \cdot)\right), n_{1}(\theta \omega)<n_{1}(\omega) .
\end{array}\right.
$$

In short, equations (2.7) are written as

$$
r \circ c=d^{\triangle} \circ d \circ r, \quad d^{\triangle} \circ r \circ c=d \circ r,
$$

where $\triangle$ denotes the adjustment that is understood as in (2.7). Similarly we interpret the diagram in (2.4). Moreover, the subscript $*$ in (2.3) and (2.4) denotes $\max \left\{n_{1}(\omega)+1, n_{1}(\theta \omega)+1\right\}$ and $\max \left\{n_{2}(\omega)+1, n_{2}(\theta \omega)+1\right\}$, respectively.

It can be proved that the shift equivalence is an equivalence relation. If $P=$ $(N(\omega), L(\omega))$ and $P^{\prime}=\left(N^{\prime}(\omega), L^{\prime}(\omega)\right)$ are two random filtration pairs for $S(\omega)$, then the induced random maps $\phi_{P}$ and $\phi_{P^{\prime}}$ on the corresponding random pointed spaces are random shift equivalent. 
Let $f$ and $g$ be Caratheodory functions. Then $f$ is called random homotopic to $g$, denoted by $f \simeq g$, if there exists a map $H:[0,1] \times \Omega \times C(\omega) \rightarrow C\left(\theta_{n} \omega\right)$ such that $H(\cdot, \omega, \cdot)$ is continuous, $H(\lambda, \cdot, x)$ is measurable, and

$$
\left\{\begin{array}{l}
H(0, \cdot, \cdot)=f(\cdot, \cdot) \\
H(1, \cdot, \cdot)=g(\cdot, \cdot)
\end{array}\right.
$$

Denote by $[f]$ the random homotopy class with $f$ the representative element. The random homotopy equivalence classes $(C,[c])$ and $(D,[d])$ are called random shift equivalent if there exist random homotopy classes $[r(\omega)]: C(\omega) \rightarrow D\left(\theta_{n_{1}} \omega\right)$ and $[s(\omega)]: D(\omega) \rightarrow C\left(\theta_{n_{2}} \omega\right)$, where $n_{i}=n_{i}(\omega)(i=1,2)$ are measurable, such that

$$
[r \circ c]=\left[d^{\triangle} \circ d \circ r\right], \quad[s \circ d]=\left[c^{\triangle} \circ c \circ s\right], \quad[r \circ s]=\left[d^{*}\right], \quad[s \circ r]=\left[c^{*}\right],
$$

where the superscript ${ }^{*}$ in the latter two equations denotes $n_{2}(\omega)+n_{1}\left(\theta_{n_{2}} \omega\right)$ and $n_{1}(\omega)+n_{2}\left(\theta_{n_{1}} \omega\right)$, respectively (see (2.5) and (2.6) $)$.

Definition 2.2 (Random Conley index 12]). Assume that $\varphi$ is the time-one map of a discrete random dynamical system, $S(\omega)$ is a random isolated invariant set for $\varphi$ and $P=(N(\omega), L(\omega))$ is a random filtration pair for $S(\omega)$. Let $h_{P}(S(\omega), \varphi)$ be the random homotopy class $\left[\varphi_{P}\right]$ on the random pointed space $N_{L}(\omega)$ with $\varphi_{P}$ a representative element. Then the random shift equivalent class of $h_{P}(S(\omega), \varphi)$, denoted by $h(S(\omega), \varphi)$, is defined as the random Conley index for $S(\omega)$.

We present a few properties of the random Conley index. These will be needed in the following sections.

Lemma 2.3. If $S_{1}(\omega)$ and $S_{2}(\omega)$ are disjoint random isolated invariant sets, then $S(\omega)=S_{1}(\omega) \cup S_{2}(\omega)$ is a random isolated invariant set.

Proof. Since $S_{1}(\omega)$ and $S_{2}(\omega)$ are random isolated invariant sets, there exist disjoint random isolating blocks $N_{1}(\omega)$ and $N_{2}(\omega)$ such that $S_{1}(\omega)=\operatorname{Inv} N_{1}(\omega)$ and $S_{2}(\omega)=\operatorname{Inv} N_{2}(\omega)$. Let $N_{1}^{\prime}(\omega):=\varphi\left(\theta_{-1} \omega, N_{1}\left(\theta_{-1} \omega\right)\right) \cap N_{1}(\omega) \cap \varphi^{-1}\left(\theta \omega, N_{1}(\theta \omega)\right)$ and $N_{2}^{\prime}(\omega):=\varphi\left(\theta_{-1} \omega, N_{2}\left(\theta_{-1} \omega\right)\right) \cap N_{2}(\omega) \cap \varphi^{-1}\left(\theta \omega, N_{2}(\theta \omega)\right)$. Then $N_{1}^{\prime}(\omega)$ and $N_{2}^{\prime}(\omega)$ are disjoint random isolating neighborhoods by the invariance of $S_{1}(\omega)$ and $S_{2}(\omega)$. We can check that $N(\omega):=N_{1}^{\prime}(\omega) \cup N_{2}^{\prime}(\omega)$ is a random isolating neighborhood for $S(\omega)$.

Lemma 2.4. If the random dynamical systems $\phi_{1}$ and $\phi_{2}$ are topologically equivalent and $S(\omega)$ is a random isolated invariant set with respect to $\phi_{1}$, then $\alpha(\omega, S(\omega))$ is a random isolated invariant set with respect to $\phi_{2}$ and

$$
h\left(S(\omega), \phi_{1}\right)=h\left(\alpha(\omega, S(\omega)), \phi_{2}\right) .
$$

Proof. Since $S(\omega)$ is a random isolated invariant set with respect to $\phi_{1}$, there exists a random isolating neighborhood $N(\omega)$ such that $S(\omega)=\operatorname{Inv} N(\omega)$. Note that $\alpha(\omega, N(\omega))$ is a random isolating neighborhood. We now show that $\alpha(\omega, S(\omega))=$ $\operatorname{Inv} \alpha(\omega, N(\omega))$. In fact, if $x \in S(\omega)$, by definition, we have $\phi_{1}(n, \omega, x) \in N\left(\theta_{n} \omega\right)$, $\forall n \in \mathbb{Z}$. So $\alpha\left(\theta_{n} \omega, \phi_{1}(n, \omega, x)\right) \in \alpha\left(\theta_{n} \omega, N\left(\theta_{n} \omega\right)\right), \forall n \in \mathbb{Z}$. That is,

$$
\phi_{2}(n, \omega, \alpha(\omega, x)) \in \alpha\left(\theta_{n} \omega, N\left(\theta_{n} \omega\right)\right) .
$$

We have $\alpha(\omega, S(\omega)) \subset \operatorname{Inv} \alpha(\omega, N(\omega))$. Similarly we can show the opposite inclusion. 
Suppose $P=(N(\omega), L(\omega))$ is a random filtration pair for $S(\omega)$. We check that $P^{\prime}=(\alpha(\omega, N(\omega)), \alpha(\omega, L(\omega)))$ is a random filtration pair for $\alpha(\omega, S(\omega))$ as follows. (i) If $x \in \operatorname{Inv} \alpha(\omega, \operatorname{cl}(N(\omega) \backslash L(\omega)))$, then there exists $y \in \operatorname{cl}(N(\omega) \backslash L(\omega))$ such that $x=\alpha(\omega, y)$ and $\phi_{2}(n, \omega, x)=\alpha\left(\theta_{n} \omega, \phi_{1}(n, \omega, y)\right) \in \alpha\left(\theta_{n} \omega, c l\left(N\left(\theta_{n} \omega\right) \backslash L\left(\theta_{n} \omega\right)\right)\right)$, and we have $\phi_{1}(n, \omega, y) \in \operatorname{cl}\left(N\left(\theta_{n} \omega\right) \backslash L\left(\theta_{n} \omega\right)\right)$. Thus $y \in \operatorname{Invcl}(N(\omega) \backslash L(\omega))$. Using the fact that $\operatorname{cl}(N(\omega) \backslash L(\omega))$ is a random isolating neighborhood of $S(\omega)$, we have $y \in S(\omega)$. Hence $\alpha(\omega, y) \in \alpha(\omega, S(\omega))$. It follows that

$$
\operatorname{Inv} \alpha(\omega, \operatorname{cl}(N(\omega) \backslash L(\omega))) \subset \alpha(\omega, S(\omega)) .
$$

Similarly we can prove that $\alpha(\omega, S(\omega)) \subset \operatorname{Inv} \alpha(\omega, \operatorname{cl}(N(\omega) \backslash L(\omega)))$. We conclude that $\alpha(\omega, c l(N(\omega) \backslash L(\omega)))$ is a random isolating neighborhood of $\alpha(\omega, S(\omega))$. (ii) Denote by $\alpha^{-}(\omega, N(\omega))$ the exit set of $\alpha(\omega, N(\omega))$. Then $\alpha^{-}(\omega, N(\omega))=\alpha\left(\omega, N^{-}(\omega)\right)$. It is easy to see that $\alpha(\omega, L(\omega))$ is a random isolating neighborhood of $\alpha^{-}(\omega, N(\omega))$. (iii) Note that

$$
\alpha\left(\theta \omega, \varphi_{1}(\omega, L(\omega))\right)=\varphi_{2}(\omega, \alpha(\omega, L(\omega)))
$$

and

$$
\varphi_{1}(\omega, L(\omega)) \cap \operatorname{cl}(N(\theta \omega) \backslash L(\theta \omega))=\emptyset .
$$

So

$$
\alpha\left(\theta \omega, \varphi_{1}(\omega, L(\omega))\right) \cap \alpha(\theta \omega, c l(N(\theta \omega) \backslash L(\theta \omega)))=\emptyset,
$$

i.e., $\varphi_{2}(\omega, \alpha(\omega, L(\omega))) \cap c l(\alpha(\theta \omega, N(\theta \omega)) \backslash \alpha(\theta \omega, L(\theta \omega)))=\emptyset$.

Denote by $\alpha_{L}(\omega, N(\omega))$ the random quotient space $\alpha(\omega, N(\omega)) / \alpha(\omega, L(\omega))$. Let $\varphi_{2 P^{\prime}}(\omega, \cdot)= \begin{cases}{[\alpha(\theta \omega, L(\theta \omega))],} & x=[\alpha(\omega, L(\omega))] \text { or } \varphi_{2}(\omega, x) \notin \alpha(\theta \omega, N(\theta \omega)), \\ p_{2}\left(\theta \omega, \varphi_{2}(\omega, x)\right), & \text { otherwise, }\end{cases}$ where $p_{2}(\omega, \cdot): \alpha(\omega, N(\omega)) \rightarrow \alpha_{L}(\omega, N(\omega))$ is the quotient map.

Consider the following two maps: $r(\omega, \cdot): N_{L}(\omega) \rightarrow \alpha_{L}(\omega, N(\omega))$,

$$
r(\omega, \cdot)= \begin{cases}{[\alpha(\omega, L(\omega))],} & x=[L(\omega)], \\ p_{2}(\omega, \alpha(\omega, x)), & \text { otherwise }\end{cases}
$$

and $s(\omega, \cdot): \alpha_{L}(\omega, N(\omega)) \rightarrow N_{L}(\omega)$,

$$
s(\omega, \cdot)= \begin{cases}{[L(\omega)],} & x=[\alpha(\omega, L(\omega))], \\ p\left(\omega, \alpha^{-1}(\omega, x)\right), & \text { otherwise }\end{cases}
$$

We verify that $r(\omega, \cdot)$ and $s(\omega, \cdot)$ are continuous and that $r(\cdot, x)$ and $s(\cdot, x)$ are measurable. Moreover we have

$$
\left[r \circ \varphi_{1 P}\right]=\left[\varphi_{2 P^{\prime}} \circ r\right],\left[s \circ \varphi_{2 P^{\prime}}\right]=\left[\varphi_{1 P} \circ s\right],[r \circ s]=[I],[s \circ r]=[I] .
$$

Thus by the definition of the random Conley index we conclude that

$$
h\left(S(\omega), \phi_{1}\right)=h\left(\alpha(\omega, S(\omega)), \phi_{2}\right) .
$$

\section{Prime Random isolated invariant SETS}

We call $S(\omega)$ a prime random isolated invariant set for $\varphi$ if $S(\omega)$ is a random isolated invariant set for $\varphi$, and for any proper subset $S^{\prime}(\omega) \subset S(\omega), S^{\prime}(\omega) \neq \emptyset$, $S^{\prime}(\omega)$ is not a random isolated invariant set for $\varphi$. Suppose $\varphi$ has only finitely many prime random isolated invariant sets $S_{1}(\omega), S_{2}(\omega), \ldots, S_{r}(\omega)$. Then $T_{f}(\omega)=$ $\bigcup_{i=1}^{r} S_{i}(\omega)$ is called an extreme maximal random isolated invariant set for $\varphi$. For the deterministic case see [6]. 
Lemma 3.1. For any two different prime random isolated invariant sets $S_{1}(\omega)$ and $S_{2}(\omega)$, we have $S_{1}(\omega) \cap S_{2}(\omega)=\emptyset$ for $\omega \in \Omega$.

Proof. Assume that $N_{1}(\omega)$ and $N_{2}(\omega)$ are random isolating neighborhoods for $S_{1}(\omega)$ and $S_{2}(\omega)$ respectively. We set $S(\omega)=S_{1}(\omega) \cap S_{2}(\omega)$ and $N(\omega)=N_{1}(\omega) \cap N_{2}(\omega)$. If $S(\omega) \neq \emptyset$, then there exists $x$ such that $x \in S(\omega)$. We have $x \in S_{i}(\omega)(i=1,2)$, which imply $\varphi^{n}(\omega, x) \in S_{1}\left(\theta_{n} \omega\right) \cap S_{2}\left(\theta_{n} \omega\right)=S\left(\theta_{n} \omega\right), n \in \mathbb{Z}$. Since $S(\omega)$ is compact and forward invariant, by the fact that $\operatorname{Inv} S(\omega)=\Omega_{S}(\omega)$, we have

$$
\operatorname{Inv} S(\omega) \neq \emptyset .
$$

It is clear that $\operatorname{InvS}(\omega) \subset \operatorname{Inv} N(\omega)$. On the other hand, for any $x \in \operatorname{Inv} N(\omega)$, since $N(\omega)=N_{1}(\omega) \cap N_{2}(\omega)$, we have $\varphi^{n}(\omega, x) \in N_{1}\left(\theta_{n} \omega\right) \cap N_{2}\left(\theta_{n} \omega\right), n \in \mathbb{Z}$, which implies $\varphi^{n}(\omega, x) \in \operatorname{Inv} N_{i}\left(\theta_{n} \omega\right)=S_{i}\left(\theta_{n} \omega\right)(i=1,2)$. It follows that $x \in \operatorname{Inv} S(\omega)$. Furthermore, we have

$$
\begin{aligned}
& \operatorname{Inv} S(\omega)=\operatorname{Inv}\left(S_{1}(\omega) \cap S_{2}(\omega)\right) \subset S_{1}(\omega) \cap S_{2}(\omega) \subset \operatorname{int} N_{1}(\omega) \cap \operatorname{int} N_{2}(\omega) \\
& =\operatorname{int}\left(N_{1}(\omega) \cap N_{2}(\omega)\right)=\operatorname{int} N(\omega) .
\end{aligned}
$$

Thus $\operatorname{Inv} S(\omega)$ is a nonempty random isolated invariant (with respect to $\varphi$ ) proper subset of $S_{i}(\omega)(i=1,2)$, which is a contradiction. So $S(\omega)=\emptyset$; i.e., $S_{1}(\omega)$ and $S_{2}(\omega)$ are disjoint.

By Lemmas 3.1 and 2.3, we see that an extreme maximal random isolated invariant set is indeed a random isolated invariant set. As in [6, if there exists no nonempty prime random isolated invariant set or if there are infinitely many prime random isolated invariant sets, then we define the extreme maximal random isolated invariant set for $\varphi$ by $T_{\varphi}(\omega)=\emptyset$. Thus, for every random homeomorphism $\varphi$, there exists a unique extreme maximal random isolated invariant set.

\section{MAin RESUlt}

Before presenting our main result, we recall the definition for abstract bifurcation points [1].

Definition 4.1 (Abstract bifurcation point). Let $\varphi_{\lambda}$ be a family of RDS on $X$, parameterized by $\lambda \in R^{k}$. A parameter value $\lambda_{0}$ is called an abstract bifurcation point of the family if the family is not structurally stable at $\lambda_{0}$, i.e., if in any neighborhood of $\lambda_{0}$ there is a parameter value $\lambda$ such that $\varphi_{\lambda}$ and $\varphi_{\lambda_{0}}$ are not topologically equivalent.

For the extreme maximal random isolated invariant set $T_{\varphi_{\lambda}}(\omega)$, we use $M\left(T_{\varphi_{\lambda}}\right)(\omega)$ to denote the cardinal number of the set $\{S(\omega) \mid S(\omega)$ is a prime random isolated invariant set for $\left.\varphi_{\lambda}\right\}$. If $T_{\varphi_{\lambda}}(\omega)=\emptyset$, we set $M\left(T_{\varphi_{\lambda}}\right)(\omega)=0$. The main result of this paper is based on the following lemma.

Lemma 4.2. Suppose $\varphi_{\lambda}$ and $\varphi_{\lambda_{0}}$ are topologically equivalent under $\alpha$. Let $T_{\varphi_{\lambda}}(\omega)$ and $T_{\varphi_{\lambda_{0}}}(\omega)$ be the extreme maximal random isolated invariant sets for $\varphi_{\lambda}$ and $\varphi_{\lambda_{0}}$, respectively. Then $M\left(T_{\varphi_{\lambda}}\right)(\omega)=M\left(T_{\varphi_{\lambda_{0}}}\right)(\omega)$ and $\alpha\left(\omega, T_{\varphi_{\lambda}}(\omega)\right)=T_{\varphi_{\lambda_{0}}}(\omega)$.

Proof. We first consider the case when $T_{\varphi_{\lambda}}(\omega) \neq \emptyset$. Then there exists an integer $r>0$ such that $T_{\varphi_{\lambda}}(\omega)=\bigcup_{i=1}^{r} S_{i}(\omega)$, where each $S_{i}(\omega)$ is a prime random isolated 
invariant set for $\varphi_{\lambda}$. Since $\varphi_{\lambda}$ and $\varphi_{\lambda_{0}}$ are topologically equivalent, by Lemma 2.4. for each $i_{0} \in\{1, \ldots, r\}, \alpha\left(\omega, S_{i_{0}}(\omega)\right)$ is an isolated invariant set of $\varphi_{\lambda_{0}}$. Suppose $\alpha\left(\omega, S_{i_{0}}(\omega)\right)$ is not a prime random isolated set; i.e., there exists a nonempty proper subset $S_{i_{0}}^{\prime}(\omega) \subset \alpha\left(\omega, S_{i_{0}}(\omega)\right)$ such that $S_{i_{0}}^{\prime}(\omega)$ is a random isolated invariant set. Then $\alpha^{-1}\left(\omega, S_{i_{0}}^{\prime}(\omega)\right) \subset S_{i_{0}}(\omega)$ is a nonempty random isolated invariant set of $\varphi_{\lambda}$, which contradicts the fact that $S_{i_{0}}(\omega)$ is a prime random isolated invariant set. Thus $\alpha$ maps a prime random isolated invariant set for $\varphi_{\lambda}$ to a prime random isolated invariant set for $\varphi_{\lambda_{0}}$. Similarly, $\alpha^{-1}$ maps a prime random isolated invariant set for $\varphi_{\lambda_{0}}$ to a prime random isolated invariant set for $\varphi_{\lambda}$. Therefore, $M\left(T_{\varphi_{\lambda}}\right)(\omega)=$ $M\left(T_{\varphi_{\lambda_{0}}}\right)(\omega)$ and $\alpha\left(\omega, T_{\varphi_{\lambda}}(\omega)\right)=\alpha\left(\omega, \bigcup_{i=1}^{r} S_{i}(\omega)\right)=\bigcup_{i=1}^{r} \alpha\left(\omega, S_{i}(\omega)\right)=T_{\varphi_{\lambda_{0}}}(\omega)$. When $T_{\varphi_{\lambda}}(\omega)=\emptyset$, we have $T_{\varphi_{\lambda_{0}}}(\omega)=\emptyset$. So we still have $\alpha\left(\omega, T_{\varphi_{\lambda}}(\omega)\right)=T_{\varphi_{\lambda_{0}}}(\omega)$.

This is our main result on bifurcation.

Theorem 4.3 (Bifurcation points for discrete-time random dynamical system). Consider a family of discrete-time $R D S \varphi_{\lambda}$, parameterized by $\lambda \in R^{k}$. If for any neighborhood $U$ containing $\lambda_{0} \in R^{k}$ there exists $\lambda \in U$ such that $M\left(T_{\varphi_{\lambda}}\right)(\omega) \neq$ $M\left(T_{\varphi_{\lambda_{0}}}\right)(\omega)$ or $h\left(T_{\varphi_{\lambda}}(\omega), \varphi_{\lambda}\right) \neq h\left(T_{\varphi_{\lambda_{0}}}(\omega), \varphi_{\lambda_{0}}\right)$, then $\lambda_{0}$ is an abstract bifurcation point of $\varphi_{\lambda}$.

Proof. Suppose $\lambda_{0}$ is not an abstract bifurcation point. Then there exists a neighborhood $U$ containing $\lambda_{0}$ such that for each $\lambda \in U$, there exists a homeomorphism $\alpha_{\lambda}$ on $X$ such that $\varphi_{\lambda}$ and $\varphi_{\lambda_{0}}$ are topologically equivalent.

From Lemma 4.2, we have $M\left(T_{\varphi_{\lambda}}\right)(\omega)=M\left(T_{\varphi_{\lambda_{0}}}\right)(\omega)$ and $\alpha_{\lambda}\left(\omega, T_{\varphi_{\lambda}}(\omega)\right)=$ $T_{\varphi_{\lambda_{0}}}(\omega)$. By Lemma 2.4, we have $h\left(T_{\varphi_{\lambda}}(\omega), \varphi_{\lambda}\right)=h\left(T_{\varphi_{\lambda_{0}}}(\omega), \varphi_{\lambda_{0}}\right)$, a contradiction. So $\lambda_{0}$ is an abstract bifurcation point of $\varphi_{\lambda}$.

Although the Conley index for continuous-time RDS (i.e., the time set $\mathbb{T}=\mathbb{R}$ ) is not available at present, we can still apply the Conley index for discrete-time RDS to detect abstract bifurcation points of continuous-time RDS. Namely, we have the following bifurcation result.

Corollary 4.4 (Bifurcation points for continuous-time RDS). Consider a family of continuous-time $R D S \varphi_{\lambda}$, parameterized by $\lambda \in R^{k}$. If for any neighborhood $U$ containing $\lambda_{0} \in R^{k}$ there exists $\lambda \in U$ such that $M\left(T_{\varphi_{\lambda}}\right)(\omega) \neq M\left(T_{\varphi_{\lambda_{0}}}\right)(\omega)$ or $h\left(T_{\varphi_{\lambda}}(\omega), \varphi_{\lambda}\right) \neq h\left(T_{\varphi_{\lambda_{0}}}(\omega), \varphi_{\lambda_{0}}\right)$ for the corresponding discrete-time $R D S$ $\varphi_{\lambda}(n, \omega, x), n \in \mathbb{Z}$, then $\lambda_{0}$ is an abstract bifurcation point for the original family of continuous-time $\operatorname{RDS} \varphi_{\lambda}$.

Proof. Note that the discrete-time $\operatorname{RDS} \varphi_{\lambda}(n, \omega, x)$ is generated via iteration by the time-one map of the continuous-time $\operatorname{RDS} \varphi_{\lambda}$. This result thus follows from Theorem 4.3 and the definition of an abstract bifurcation point.

We present two examples to demonstrate the above bifurcation results for RDS.

Example 4.5 (Bifrucation in a discrete-time RDS). Consider a family of discretetime $\operatorname{RDS} \varphi_{\lambda}$ on $\mathbb{R}$,

$$
\varphi_{\lambda}(\omega, x)= \begin{cases}x+x^{2}+\lambda \xi(\omega), & x \geq-\frac{1}{2} \\ -\frac{1}{2} x+\lambda \xi(\omega), & x<-\frac{1}{2}\end{cases}
$$


where $\lambda \in \mathbb{R}$ is a real parameter and $\xi(\omega)$ is a given positive random variable. There exists a prime random isolated invariant set for $\lambda \leq 0$. But for $\lambda>0$, there is no such prime random isolated invariant set. So $\lambda=0$ is an abstract bifurcation point for the discrete-time $\operatorname{RDS} \varphi_{\lambda}$ by Theorem 4.3 .

We revise an example from [14 to fit our purpose here.

Example 4.6 (Bifrucation in a continuous-time RDS). Consider a family of scalar random differential equations

$$
x^{\prime}=f_{\lambda}\left(x, \theta_{t} \omega\right)
$$

parameterized by a real parameter $\lambda \in[-1,1]$. Assume that (4.1) generates a family of $\operatorname{RDS} \varphi_{\lambda}(t, \omega, x)$ and that there exists a unique fixed point $0,|\lambda| \leq 1$. Denote the corresponding discrete-time RDS by $\varphi_{\lambda}(n, \omega, x)$. From Theorem 7.2 of 12 we know that $\{0\}$ is the random isolated invariant set of $\varphi_{\lambda}(n, \omega, x)$. If $\lim _{n \rightarrow \pm \infty} \varphi_{\lambda}(n, \omega, x)=0$ for $\lambda>0, x \in \mathbb{R}$, and $\lim _{n \rightarrow+\infty} \varphi_{\lambda}(n, \omega, x)=0, \lim _{n \rightarrow-\infty} \varphi_{\lambda}(n, \omega, x)=-\infty$ for $\lambda<0$, $x \in \mathbb{R}$. Let $\underline{0}$ be the random Conley index of random pointed spaces consisting of just one random point with random constant maps as their corresponding random pointed space maps. Then the Conley index of $\{0\}$ for $\lambda>0$ is not equal to $\underline{0}$, but the Conley index of $\{0\}$ for $\lambda<0$ is $\underline{0}$. We can conclude that $\lambda=0$ is the abstract bifurcation point of continuous-time $\operatorname{RDS} \varphi_{\lambda}$ by Corollary 4.4 .

\section{REFERENCES}

1. L. Arnold, Random Dynamical Systems, Springer, Berlin-Heidelberg-New York, 1998. MR.1723992 (2000m:37087)

2. T. Bartsch, The Conley Index over a Space, Mathematische Zeitschrift 209(1992), 167-177. MR.1147812 (92m:58019)

3. I. Chueshov, Monotone Dynamical Systems Theory and Applications, Springer Lecture Notes in Mathematics, vol. 1779, Berlin-Heidelberg-New York, 2002. MR1902500 (2003d:37072)

4. C. Conley, Isolated Invariant Sets and the Morse Index, Conf. Board Math. Sci., vol. 38, Amer. Math. Soc., Providence, RI, 1978. MR.511133 (80c:58009)

5. J. Franks and D. Richeson, Shift Equivalence and the Conley Index, Transactions of the American Mathematical Society 352(2000), 3305-3322. MR 1665329 (2000j:37013)

6. X. C. Fu and K. H. Xu, The Conley Index and Bifurcation Points, Nonlinear Analysis: Theory, Methods \& Applications 19(1992), 1137-1142. MR.1195047 (93j:58096)

7. M. Izydorek and S. Rybicki, Bifurcations of Bounded Solutions of 1-Parameter ODE's, Journal of Differential Equations 130(1996), 267-276. MR.1410887 (97i:34053)

8. E. Kappos, The Conley Index and Global Bifurcations, Part I: Concepts and Theory, International Journal of Bifurcation and Chaos 5(1995), 937-953. MR.1348294 (96f:58112)

9. E. Kappos, The Conley Index and Global Bifurcations. II. Illustrative Applications, International Journal of Bifurcation and Chaos 6(1996), 2491-2505. MR1450272 (98d:58129)

10. P. Krzysztof and Rybakowski, The Homotopy Index and Partial Differential Equations, Springer, Berlin-Heidelberg-New York, 1987. MR910097 (89d:58025)

11. M. Kunze, Non-Smooth Dynamical Systems, Springer Lecture Notes in Mathematics, vol. 1744, Berlin-Heidelberg-New York, 2000. MR.1789550 (2002e:34002)

12. Z. X. Liu, Conley Index for Random Dynamical Systems, Journal of Differential Equations 244(2008), 1603-1628. MR2404433 (2009g:37048) 
13. K. Mischaikow and M. Mrozek, Conley Index. Handbook of dynamical systems, vol. 2, 393460, North-Holland, Amsterdam, 2002. MR.1901060 (2003g:37022)

14. J. Smoller, Shock Waves and Reaction-Diffusion Equations, Springer, Berlin-HeidelbergNew York, 1983. MR688146 (84d:35002)

School of Mathematics and Statistics, Huazhong University of Science and TechNOLOGY, WuHAN 430074, China

E-mail address: chenxiao002214336@yahoo.cn

Department of Applied Mathematics, Illinois Institute of Technology, Chicago, IlliNOIS 60616

E-mail address: duan@iit.edu

Department of Mathematics, Shanghai University, Shanghai 200444, People's RepubLIC OF CHINA

E-mail address: xcfu@shu.edu.cn 